\title{
Probing Chemical Short-range Order and other Nanoscale Motifs in the Solid State
}

\author{
Katharine Page \\ University of Tennessee, Knoxville, United States of America; \\ kpage10@utk.edu
}

Next generation materials of nearly every kind rely on chemical, electronic, and/or magnetic heterogeneity for creating, harnessing, and controlling functionality. Exploration of these phenomena increasingly involve multiple length-scale scattering probes and require sophisticated modeling approaches to characterize and understand them. Total scattering methods, including both Bragg and diffuse scattering signals, are providing key insights into how long-range, nanoscale, and local atomic structure motifs differ in materials and cooperate to deliver their unique properties. The nuances of capturing nanoscale heterogeneities, including correlated defects, chemical short-range order, and stacking fault distributions, represent a modern frontier in the field of crystallography. We will explore this theme through detailed investigation of two distinct materials classes. First, we will present the operando study of nanostructured fluorite catalysts. We will specifically follow the nature of correlated oxygen vacancies at elevated temperatures, including their behavior under acid-gas exposure. Second, we will present structure-property characteristics of new pyrochlore and perovskite high entropy oxides (HEOs). HEOs exhibit a single-phase crystal structure containing five or more different metal cations of the same amount on single crystallographic lattice sites; their compositional and configurational disorder and associated structural diversity offer great potential for unique material characteristics. We will highlight contemporary challenges and opportunities in the quest to extract crystal structure models from experimental data with the detail needed to guide and validate solid state theories, and design new and improved functional materials.

Keywords: x-ray diffraction, neutron diffraction, pair distribution function, catalysts, complex oxides 